\title{
Relationship between the extent of central node dissection and parathyroid function preservation in thyroid cancer surgery
}

\author{
Ronghao Sun ${ }^{1,2 \#}$, Jianfeng Sheng ${ }^{3 \#}$, Yue Zhou ${ }^{4}$, Yuqiu Zhou ${ }^{1}$, Yongcong Cai ${ }^{1}$, Chunyan Shui ${ }^{1}$, \\ Dingfen Zeng ${ }^{1}$, Jian Jiang', Rui Li ${ }^{1}$, Xu Wang ${ }^{1}$, Jingqiang Zhu ${ }^{2}$, Chao Li ${ }^{1}$
}

${ }^{1}$ Department of Head and Neck Surgery, Sichuan Cancer Hospital and Institute, Sichuan Cancer Center, School of Medicine, University of Electronic Science and Technology of China, Chengdu, China; ${ }^{2}$ Department of Thyroid \& Parathyroid Surgery, West China Hospital, Sichuan University, Chengdu, China; ${ }^{3}$ Department of Otorhinolaryngology Head and Neck Surgery, Mianyang Third People's Hospital, Mianyang, China; ${ }^{4}$ Department of Radiotherapy, Hunan Cancer Hospital and the Affiliated Cancer Hospital of Xiangya School of Medicine, Central South University, Changsha, China

Contributions: (I) Conception and design: R Sun, C Li, J Sheng, Y Zhou; (II) Administrative support: R Sun, C Li, Y Cai; (III) Provision of study materials or patients: X Wang, J Jiang, D Zeng, C Li; (IV) Collection and assembly of data: R Sun, C Shui; (V) Data analysis and interpretation: Y Cai, R Sun, R Li; (VI) Manuscript writing: All authors; (VII) Final approval of manuscript: All authors.

\#These authors contributed equally to this work.

Correspondence to: Chao Li. Department of Head and Neck Surgery, Sichuan Cancer Hospital and Institute, Sichuan Cancer Center, School of Medicine, University of Electronic Science and Technology of China, Chengdu 610041, China. Email: headneck@qq.com; Jingqiang Zhu. Department of Thyroid \& Parathyroid Surgery, West China Hospital, Sichuan University, Chengdu 610000, China. Email: zjq-wkys@163.com.

Background: The risk and effect of hypocalcemia following surgery of different magnitudes remains unclear. Thus, we assessed whether different extents of central lymph node dissection (CLND) and status of preserved parathyroid glands can influence parathyroid function in differentiated thyroid carcinoma (DTC) patients with initial surgical resection.

Methods: Participants were categorized into 6 groups based on the extent of the surgical procedures, number of parathyroid glands preserved in situ, and parathyroid autotransplantation. The frequencies of hypocalcemia, serum calcium (Ca) levels, and parathyroid hormone (PTH) levels among the different groups were analyzed.

Results: The prevalence of hypocalcemia, number and status of parathyroid glands preserved in situ, and parathyroid autotransplantation were inversely related to extensive CLND ( $\mathrm{r}=-0.18 ; \mathrm{P}<0.05)$. The decrease of serum $\mathrm{Ca}$ and PTH was most severe on postoperative days (POD) 1-7. The incidence of hypocalcemia was higher in Group $\mathrm{C}$ than in Group B and $\mathrm{A}(\mathrm{P}<0.05)$. The average postoperative serum $\mathrm{Ca}$ and PTH levels in Group C were significantly lower than group A on POD 1-7 $(\mathrm{P}<0.05)$. The incidence of hypocalcemia was obviously increased in Group $\mathrm{D}$ compared to Groups $\mathrm{E}$ and $\mathrm{F}(\mathrm{P}<0.05)$. The mean serum $\mathrm{Ca}$ and PTH levels in Group D were significantly lower than in Group F $(\mathrm{P}<0.05)$, and the same results could be observed between Groups D and $\mathrm{E}(\mathrm{P}<0.05)$. However, through parathyroid autotransplantation, there was no significant difference that could be found between Groups E and F on POD 1-7 (P>0.05).

Conclusions: With the expansion of CLND scope, postoperative parathyroid function will be affected, increasing the risk of postoperative hypocalcemia. When at least 1-2 parathyroid glands were reserved in situ plus at least 1 parathyroid gland autotransplantation, there was little effect on postoperative parathyroid function.

Koywords: Differentiated thyroid carcinoma (DTC); central lymph node dissection (CLND); parathyroid glands; hypocalcemia

Submitted Jan 05, 2021. Accepted for publication Mar 22, 2021.

doi: $10.21037 /$ gs-21-121

View this article at: http://dx.doi.org/10.21037/gs-21-121 


\section{Introduction}

Hypocalcemia following operation for differentiated thyroid carcinoma (DTC) remains one of the most common postoperative complications, it may prolong hospitalization and require medical intervention (1). A series of studies have reported incidences of $20-80 \%(2,3)$. Hypocalcemia is well recognized to associate with the parathyroid gland injury during thyroid surgery. The common causes of intraoperative parathyroid injury are inadvertent damage or removal of the parathyroid glands, ligation or interruption of the blood supply, and so on (4).

A surgical strategy designed to preserve sufficient numbers of parathyroid glands is traditionally advocated, which is critical for preventing clinical or symptomatic hypocalcemia. Many methods have been used to protect the parathyroid glands $(5,6)$; however, how many parathyroid glands are needed to effectively reduce postoperative hypocalcemia is always a hot topic for specialists. Some studies have supported that the preservation of all parathyroid glands decreased the risk of hypocalcemia in comparison to preservation of 3 or fewer glands, although the authors believed that routine identification of all 4 parathyroid glands was unnecessary (7). Several studies have shown that the incidence of hypocalcemia was reduced with preservation of at least one parathyroid gland with intact blood supply (8). Some scholars have suggested that the functional recovery of superior and inferior parathyroids preserved in situ is different (9). In addition, some scholars have indicated that parathyroid autotransplantation is another effective strategy to preserve parathyroid function (10).

More extensive neck dissection, especially in the central level, may increase the likelihood of sacrificing or damaging the parathyroid glands and blood supply, which is associated with higher risk of hypocalcemia (11). Moreover, the procedure of central lymph node ( $\mathrm{LN}$ ) dissection may affect the difficulty and 1 of parathyroid preservation in different parts and different states. Therefore, some research teams have established a new parathyroid classification method for intraoperative evaluation of parathyroid preservation in situ or autotransplantation (12). Now, increasing numbers surgeons have begun to heed to the influence of central lymph node dissection (CLND) on postoperative parathyroid function, and CLND is being applied more cautiously. The indications of preventive central lymph node dissection (pCLND) remain a hot topic today (13).

Because of these ambiguous clinical issues, the purpose of this study was to explore the effect of different CLND on parathyroid function preservation. Concurrently, we evaluated the predictive value of different numbers of parathyroid glands preserved in situ and autotransplanted on postoperative hypocalcemia. We present the following article in accordance with the STROBE reporting checklist (available at http://dx.doi.org/10.21037/gs-21-121).

\section{Methods}

\section{Participant characteristics}

This was an internal Institutional Review Board-approved retrospective study. Between January 2010 and January 2020, a total of 435 patients with previously untreated DTC underwent surgery as first-line therapy at the Sichuan Cancer Hospital, Sichuan, China. Participants' characteristics, surgical extents, thyroid tumor location, tumor size, lymph node metastases (LNM), number of parathyroid glands preserved, incidence of hypocalcemia, relevant serological parameters, and other clinicopathologic characteristics were recorded. Exclusion criteria included distant metastases at diagnoses, history of prior head and neck cancer, or other thyroid malignancies. Patients who had severe systemic disease, abnormal preoperative parathyroid tests, any diseases that interfered with calcium (Ca) homeostasis, or incomplete data were also excluded.

\section{Surgical procedures and parathyroid preservation}

Total thyroidectomy (TT) was performed alongside selective CLND to selected patients. A total of 150 participants underwent unilateral CLND, while bilateral CLND was performed on 189 patients (Figure 1). Participants were categorized based on the surgical procedures. Group A underwent TT plus anterior laryngeal and trachea LND only. Group B underwent TT + unilateral tracheoesophageal groove LND, and group $\mathrm{C}$ underwent TT + bilateral tracheoesophageal groove LND.

During the surgery, meticulous extracapsular dissection was made in order to identify parathyroid glands and preserve the supplying blood vessels. Terminal branches of the superior and inferior thyroid arteries were ligated distally as close to the thyroid glands as possible. The macroscopic appearance of the preserved parathyroid glands was grouped as following: D situation: 1-2 parathyroid preservation in situ without parathyroid implantation; E situation: 1-2 parathyroid preservation in situ plus at least 1 parathyroid implantation; F situation: 3-4 parathyroid 

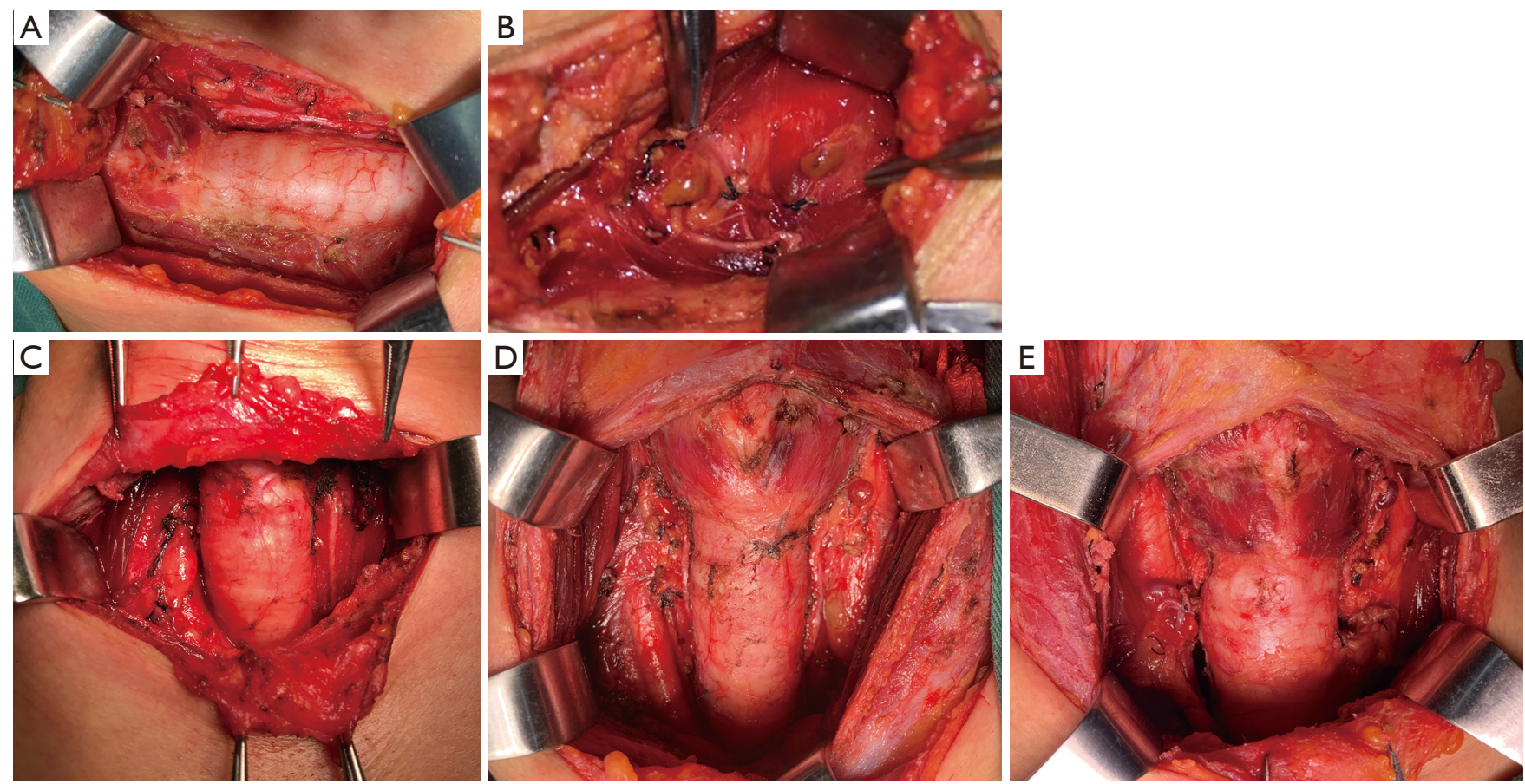

Figure 1 Hypocalcemia associated with surgical extent and the number of parathyroid glands preserved. (A) TT+ unilateral CLND: 2 intact parathyroid being preserved; (B) TT+ unilateral:2 parathyroid glands being preserved without blood supply and the color changed; (C) TT + bilateral CLND with 1 intact parathyroid gland being preserved; (D) TT + bilateral CLND with 2 intact parathyroid gland being preserved and 1 parathyroid glands were preserved without blood supply and the color changed; (E) TT + bilateral CLND with 3 intact parathyroid were preserved combined with blood supply preservation and the color was normal, but 1 parathyroid glands preserved without blood supply and the color changed. The arrow shows parathyroid gland. CLND, central lymph node dissection.

preservation in situ plus at least 1 parathyroid implantation. All data were recorded in the operative notes. We used sternocleidomastoid or anterior cervical banded muscle for intramuscular autoimplantation of parathyroid glands with poor blood supply and free parathyroid glands. The specific method used was homogenate injection after grinding.

\section{Perioperative evaluation}

Serum Ca and parathyroid hormone levels were measured by standard methods. The serum calcium and parathyroid hormone (PTH) levels were collected on the day preoperative, 1-7 days postoperative, and more than 7 days, respectively. Symptomatic hypocalcemia was defined with at least 1 of following symptoms: tingling, numbness, or carpopedal spasm along with hypocalcemia.

\section{Statistical analysis}

The statistical software SPSS version 17.0 (IBM Corp.,
Chicago, IL, USA) was used to analyze the data. Continuous data were represented as mean $\pm \mathrm{SD}$ and compared using variance analysis. Categorical variables were evaluated using a chi-square or Fisher's exact test as appropriate. To evaluate the impact of different surgical extents on the rate of postoperative hypocalcemia and other relevant serological parameters, participants were classified into groups $\mathrm{A}, \mathrm{B}$, and C. To assess the effect of preserved parathyroid glands on the study variables above, participants were also categorized into groups D, E, and F. Correlation analysis of categorical variables was conducted to establish the relationship between different surgical extents and parathyroid glands preserved. A P value $<0.05$ was considered statistically significant.

\section{Ethical statement}

All procedures performed in this study involving human participants were in accordance with the Declaration of Helsinki (as revised in 2013). The study was reviewed and approved by the Ethics committee of Sichuan Cancer Hospital. 
Table 1 Demographic and clinicopathological characteristics of 435 patients with DTC

\begin{tabular}{|c|c|}
\hline Variables & No. of patients (\%) \\
\hline \multicolumn{2}{|l|}{ Gender } \\
\hline Male & $111(25.5)$ \\
\hline Female & $324(74.5)$ \\
\hline Age, mean (SD), years & $42.94(13.44)$ \\
\hline \multicolumn{2}{|l|}{ Pathology } \\
\hline Papillary & $406(93.3)$ \\
\hline Follicular & $29(6.7)$ \\
\hline Tumor size, mean (SD), cm & $1.89(1.33)$ \\
\hline \multicolumn{2}{|l|}{ Tumor location } \\
\hline Upper pole & $145(33.4)$ \\
\hline Middle & $158(36.3)$ \\
\hline Lower pole & $132(30.3)$ \\
\hline \multicolumn{2}{|l|}{ Multifocality } \\
\hline Yes & $211(48.5)$ \\
\hline No & $224(51.5)$ \\
\hline \multicolumn{2}{|l|}{ Affected lobes } \\
\hline Unilateral & $311(71.5)$ \\
\hline Bilateral & $124(28.5)$ \\
\hline \multicolumn{2}{|l|}{ Capsular invasion } \\
\hline Yes & $201(46.2)$ \\
\hline No & $234(53.8)$ \\
\hline \multicolumn{2}{|l|}{ Central LNM } \\
\hline Yes & $254(58.4)$ \\
\hline No & $181(41.6)$ \\
\hline \multicolumn{2}{|l|}{ Surgical extent } \\
\hline$A$ & $96(22.1)$ \\
\hline $\mathrm{B}$ & $150(34.5)$ \\
\hline C & $189(43.4)$ \\
\hline \multicolumn{2}{|l|}{ Hypocalcemia } \\
\hline Normal & $226(51.9)$ \\
\hline Asymptomatic & $110(25.3)$ \\
\hline Symptomatic & $99(22.8)$ \\
\hline
\end{tabular}

Table 1 (continued)
Table 1 (continued)

\begin{tabular}{lc}
\hline Variables & No. of patients (\%) \\
\hline Parathyroid glands preserved & \\
D & $95(21.8)$ \\
E & $161(37.0)$ \\
F & $179(41.1)$ \\
\hline
\end{tabular}

A, TT + anterior laryngeal and trachea LND only; B, TT + unilateral tracheoesophageal groove LND; C, TT + bilateral tracheoesophageal groove LND; D, 1-2 parathyroid preservation in situ without parathyroid autotransplantation; E, 1-2 parathyroid preservation in situ + at least 1 parathyroid autotransplantation; $\mathrm{F}, 3-4$ parathyroid preservation in situ + at least 1 parathyroid autotransplantation. LNM, lymph node metastasis; LND, lymph node dissection; TT, total thyroidectomy; DTC, differentiated thyroid cancer.

Individual consent for this retrospective analysis was waived.

\section{Results}

\section{Demographic characteristics}

Participant demographic characteristics are shown in Table 1. Participants were grouped by the CLND extent: Group A ( $\mathrm{n}=96,22.1 \%)$; Group B ( $\mathrm{n}=50,34.5 \%)$; Group C ( $\mathrm{n}=189$, 43.4\%). All participants were also classified based on the status of parathyroid preserved: Group D ( $\mathrm{n}=95,21.8 \%)$, Group E ( $\mathrm{n}=161,37.0 \%)$, Group F ( $\mathrm{n}=179,41.1 \%)$. Totally, 209 participants were diagnosed with postoperative hypocalcemia, $110(52.6 \%)$ were asymptomatic, while 99 (47.4\%) demonstrated clinically symptomatic hypocalcemia. Among the total of 435 study participants, 226 (51.9\%) had normal postoperative serum Ca levels without any clinical hypocalcemia symptoms.

\section{Correlations of hypocalcemia, serum $\mathrm{Ca}$, and $\mathrm{PTH}$ with extent of surgical procedures}

The correlations of hypocalcemia, serum $\mathrm{Ca}$, and PTH with extent of surgical procedures is shown in Table 2. The comparisons of normal, asymptomatic, and symptomatic hypocalcemia, are demonstrated in Figure $2 \mathrm{~A}$, which shows a tendency for high risk of hypocalcemia associated with extensive surgical extents. In Group A, we observed 
Table 2 Hypocalcemia and relevant serological parameters associated with surgical extent

\begin{tabular}{|c|c|c|c|c|c|c|}
\hline Variable & \multicolumn{3}{|c|}{ Surgical extent } & \multicolumn{3}{|c|}{$P$ value } \\
\hline Hypocalcemia, n (\%) & & & & $<0.05$ & 0.04 & $<0.05$ \\
\hline Normal & $62(64.6)$ & $81(54.0)$ & $83(43.9)$ & & & \\
\hline Asymptomatic & $25(26.0)$ & $28(18.7)$ & $57(30.2)$ & & & \\
\hline \multicolumn{7}{|c|}{ Serum calcium $(\mathrm{mmol} / \mathrm{L})$, mean $\pm \mathrm{SD}$} \\
\hline POD 0 & $2.39 \pm 0.14$ & $2.40 \pm 0.13$ & $2.38 \pm 0.13$ & 0.34 & 0.18 & 0.87 \\
\hline POD 1-7 & $2.08 \pm 0.24$ & $2.03 \pm 0.28$ & $2.00 \pm 0.26$ & 0.09 & 0.45 & 0.02 \\
\hline $\mathrm{POD}>7$ & $2.24 \pm 0.13$ & $2.20 \pm 0.15$ & $2.13 \pm 0.19$ & 0.06 & $<0.05$ & $<0.05$ \\
\hline POD 1-7 & $23.88 \pm 9.74$ & $22.56 \pm 13.09$ & $18.81 \pm 11.40$ & 0.39 & 0.003 & 0.001 \\
\hline $\mathrm{POD}>7$ & $29.74 \pm 10.72$ & $28.76 \pm 11.76$ & $25.67 \pm 12.25$ & 0.53 & 0.02 & 0.006 \\
\hline Total & 96 & 150 & 189 & & & \\
\hline
\end{tabular}

A, TT plus anterior laryngeal and trachea LND only; B, TT + unilateral tracheoesophageal groove LND; C, TT + bilateral tracheoesophageal groove LND. PTH, parathyroid hormone; POD, postoperative day.
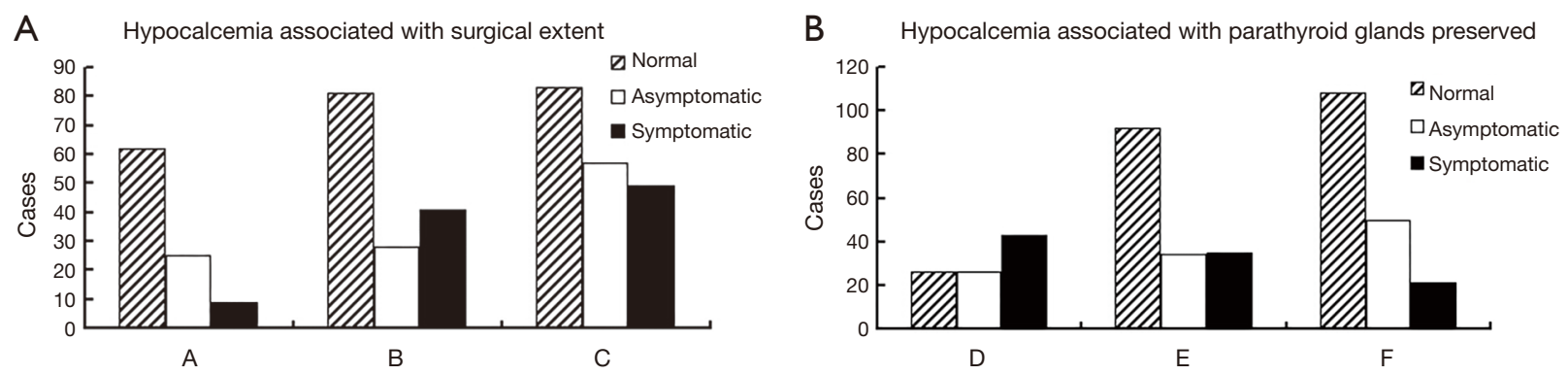

Figure 2 Hypocalcemia associated with surgical extent (A) and number of parathyroid preserved in situ and implantation (B). (A) TT plus anterior laryngeal and trachea LND only; (B) TT + unilateral tracheoesophageal groove LND; (C) TT + bilateral tracheoesophageal groove LND; (D) 1-2 parathyroid preservation in situ without parathyroid autotransplantation; (E) 1-2 parathyroid preservation in situ plus at least one parathyroid autotransplantation; (F) 3-4 parathyroid preservation in situ plus at least one parathyroid autotransplantation. TT, total thyroidectomy; LND, lymph node dissection.

$25(26.0 \%)$ asymptomatic and $9(9.4 \%)$ symptomatic participants among the cases of hypocalcemia. In group $\mathrm{B}$, the number of asymptomatic hypocalcemia cases was $28(18.7 \%)$, which was slightly lower than that of group A, while the number of symptomatic hypocalcemia cases in group B was 41 (27.3\%), which was significantly higher than that of group A. Both asymptomatic and symptomatic hypocalcemia rates were highest in group $\mathrm{C}$, which were
$57(30.2 \%)$ and $49(25.9 \%)$, respectively, among the 3 groups. The difference among the 3 groups was statistically significant (A vs. $\mathrm{B}, \mathrm{P}<0.05$; $\mathrm{B}$ vs. $\mathrm{C}, \mathrm{P}=0.04$; $\mathrm{A}$ vs. $\mathrm{C}$, $\mathrm{P}<0.05)$. There were tendencies that the more parathyroid preserved in situ and appropriate parathyroid implantation, the fewer cases of asymptomatic or symptomatic hypocalcemia (Figure 2B). There were $26(27.4 \%$ ) asymptomatic and $43(45.2 \%)$ symptomatic hypocalcemia 

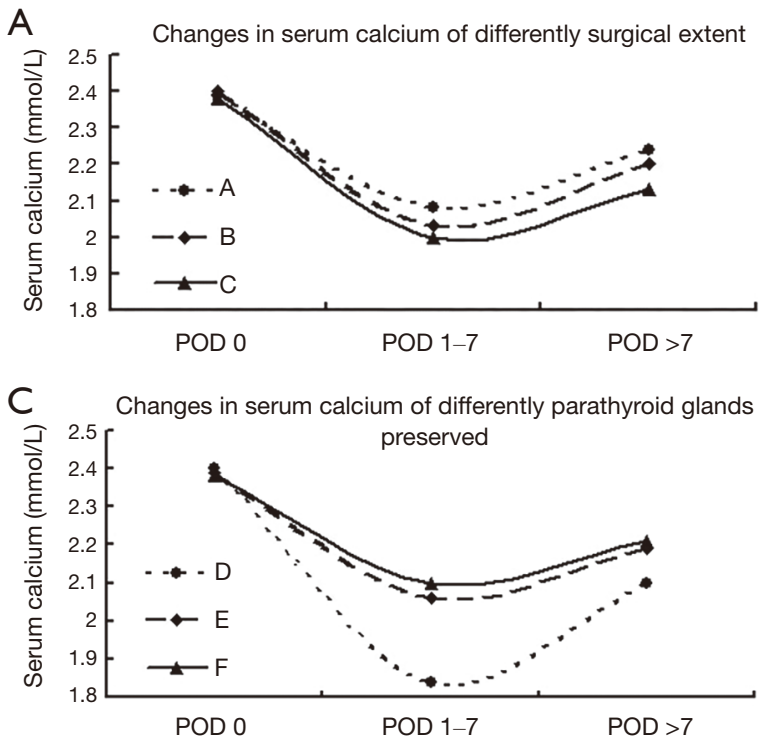

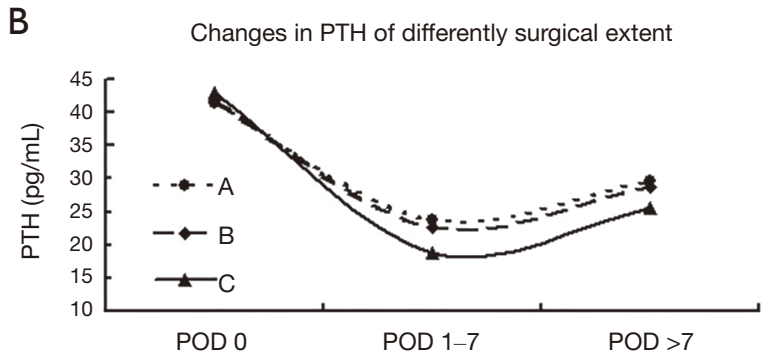

D Changes in PTH of differently parathyroid glands preserved

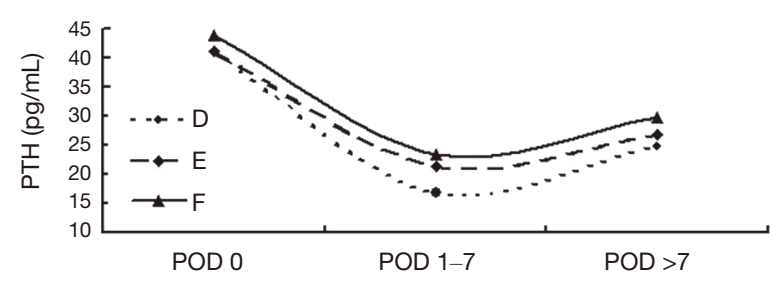

Figure 3 Changes in serum calcium and PTH of different surgical extent $(\mathrm{A}, \mathrm{B})$ and number of parathyroid preserved in situ and autotransplantation (C,D). (A) TT + anterior laryngeal and trachea LND only; (B) TT + unilateral tracheoesophageal groove LND; (C) TT + bilateral tracheoesophageal groove LND; (D) 1-2 parathyroid preservation in situ without parathyroid autotransplantation; (E) 1-2 parathyroid preservation in situ + at least 1 parathyroid autotransplantation; (F) 3-4 parathyroid preservation in situ + at least 1 parathyroid autotransplantation. PTH, parathyroid hormone; POD, postoperative day; TT, total thyroidectomy; LND, lymph node dissection.

cases observed in group D. In group E, asymptomatic and symptomatic hypocalcemia cases were $34(21.1 \%)$ and $35(21.7 \%)$, respectively, which were slightly lower than those of group D. The numbers of asymptomatic and symptomatic indexes were highest and lowest in group F, which were $50(27.9 \%)$ and $21(11.8 \%)$, respectively. The difference among the 3 groups was statistically significant (D vs. $\mathrm{E}, \mathrm{P}<0.05 ; \mathrm{E} v s . \mathrm{F}, \mathrm{P}=0.03 ; \mathrm{D}$ vs. $\mathrm{F}, \mathrm{P}<0.05$ ). These results demonstrated that intraoperative preservation of more parathyroid glands and appropriate parathyroid implantation could reduce the risk of hypocalcemia.

Our results revealed that unilateral and bilateral CLND may increase the possibility of hypocalcemia after DTC surgery. As shown in Figure $3 A$, B, variations of perioperative serum calcium and PTH both decreased on postoperative days 1-7 (POD 1-7) firstly, and recovered after more than 7 postoperative days (POD >7). However, the degree of these variations became more pronounced with more extensive CLND. As shown in Table 2, during the perioperative period, there was a slight but statistically significant difference in mean serum Ca and PTH. During POD 1-7, the mean level of serum $\mathrm{Ca}$ in group $\mathrm{C}(2.00 \pm 0.26 \mathrm{mmol} / \mathrm{L})$ was significantly lower than that of group A $(2.08 \pm 0.24 \mathrm{mmol} / \mathrm{L})(\mathrm{P}=0.02)$, and the levels of PTH in group B $(22.6 \pm 13.1 \mathrm{pg} / \mathrm{mL})$ and group A $(23.9 \pm 9.7 \mathrm{pg} / \mathrm{mL})$ was significantly higher than that of group C $(18.8 \pm 11.4 \mathrm{pg} / \mathrm{mL})$, respectively (all $\mathrm{P}<0.05)$. When POD $>7$, the levels of either serum $\mathrm{Ca}$ or $\mathrm{PTH}$ were significantly lower in group C $(2.13 \pm 0.19 \mathrm{mmol} / \mathrm{L}$ and $25.7 \pm 12.3 \mathrm{pg} / \mathrm{mL})$ than group B $(2.20 \pm 0.15 \mathrm{mmol} / \mathrm{L}$ and $28.8 \pm 11.8 \mathrm{pg} / \mathrm{mL})$ and group $\mathrm{A}(2.24 \pm 0.13 \mathrm{mmol} / \mathrm{L}$ and $29.7 \pm 10.7 \mathrm{pg} / \mathrm{mL}$ ), respectively (all $\mathrm{P}<0.05$ ). Nevertheless, Table 2 shows that the values of serum Ca and PTH had no statistical differences between groups $\mathrm{A}$ and $\mathrm{B}$ either during either POD 1-7 or POD $>7(\mathrm{P}>0.05)$.

\section{Correlations of hypocalcemia, serum Ca and PTH with the number and status of parathyroid glands preserved}

Table 3 shows the correlations of hypocalcemia, serum Ca and PTH with the number and status of parathyroid glands preserved. The changes of perioperative serum $\mathrm{Ca}$ and PTH significantly reduced during POD $1-7$, and then gradually increased after POD $>7$ (Figure 3C,D). However, the degree of these changes became more noticeable alongside fewer preserved parathyroid glands and less blood supply. As shown in Table 3, during POD 1-7, the mean levels of either serum Ca or PTH were significantly lower $(\mathrm{P}<0.05)$ in group $\mathrm{D}$ $(1.84 \pm 0.35 \mathrm{mmol} / \mathrm{L}$ and $16.8 \pm 9.3 \mathrm{pg} / \mathrm{mL})$ than in group 
Table 3 Hypocalcemia and relevant serological parameters associated with the number of parathyroid preserved in situ and implantation

\begin{tabular}{|c|c|c|c|c|c|c|}
\hline Variable & \multicolumn{3}{|c|}{ Parathyroid preserved and autotransplantation } & \multicolumn{3}{|c|}{$P$ value } \\
\hline Hypocalcemia, n (\%) & & & & $<0.05$ & 0.032 & $<0.05$ \\
\hline Normal & $26(27.4)$ & $92(57.2)$ & $108(60.3)$ & & & \\
\hline Asymptomatic & $26(27.4)$ & $34(21.1)$ & $50(27.9)$ & & & \\
\hline \multicolumn{7}{|c|}{ Serum calcium (mmol/L), mean \pm SD } \\
\hline POD 0 & $2.40 \pm 0.12$ & $2.39 \pm 0.15$ & $2.38 \pm 0.11$ & 0.85 & 0.47 & 0.41 \\
\hline POD 1-7 & $1.84 \pm 0.35$ & $2.06 \pm 0.24$ & $2.10 \pm 0.17$ & $<0.05$ & 0.12 & $<0.05$ \\
\hline $\mathrm{POD}>7$ & $2.10 \pm 0.19$ & $2.19 \pm 0.18$ & $2.21 \pm 0.15$ & $<0.05$ & 0.26 & $<0.05$ \\
\hline POD 1-7 & $16.75 \pm 9.29$ & $21.32 \pm 11.46$ & $23.50 \pm 12.78$ & 0.002 & 0.084 & $<0.05$ \\
\hline $\mathrm{POD}>7$ & $24.75 \pm 12.99$ & $26.88 \pm 10.89$ & $29.84 \pm 11.73$ & 0.16 & 0.02 & 0.001 \\
\hline Total & 95 & 161 & 179 & & & \\
\hline
\end{tabular}

D, 1-2 parathyroid preservation in situ without parathyroid autotransplantation; E, 1-2 parathyroid preservation in situ + at least 1 parathyroid autotransplantation; F, 3-4 parathyroid preservation in situ + at least 1 parathyroid autotransplantation. PTH, parathyroid hormone; POD, postoperative day.

Table 4 Co-relationship between surgical extent and the status of parathyroid preserved

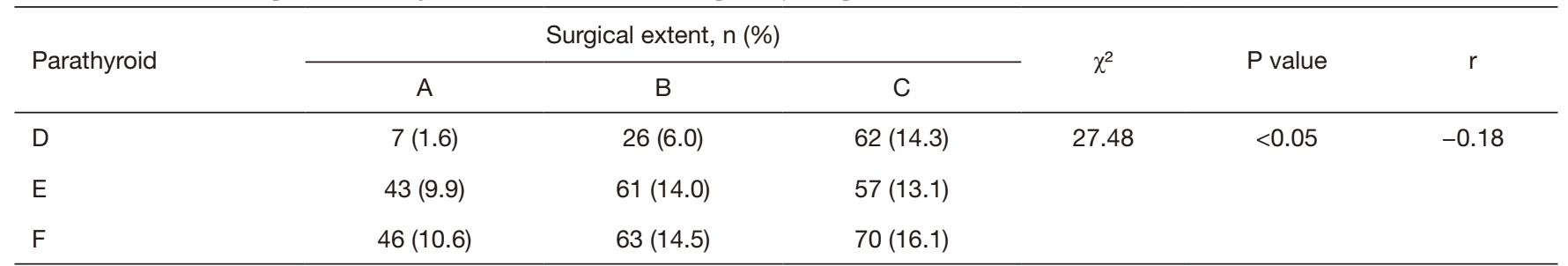

A, TT + anterior laryngeal and trachea LND only; B, TT + unilateral tracheoesophageal groove LND; C, TT + bilateral tracheoesophageal groove LND; D, 1-2 parathyroid preservation in situ without parathyroid autotransplantation; E, 1-2 parathyroid preservation in situ + at least 1 parathyroid autotransplantation; F, 3-4 parathyroid preservation in situ + at least 1 parathyroid autotransplantation. TT, total thyroidectomy; LND, lymph node dissection.

E $(2.06 \pm 0.24 \mathrm{mmol} / \mathrm{L}$ and $21.3 \pm 11.5 \mathrm{pg} / \mathrm{mL})$ and group $\mathrm{F}$ $(2.10 \pm 0.17 \mathrm{mmol} / \mathrm{L}$ and $23.5 \pm 12.8 \mathrm{pg} / \mathrm{mL})$, which had the highest levels of these 2 measurements. However, there were no significant differences in mean values of both serum $\mathrm{Ca}$ and PTH at POD $0(\mathrm{P}>0.05)$.

\section{Correlation between surgical extent and preservation of parathyroid glands}

The correlation between surgical extent and preservation of parathyroid glands is shown in Table 4. As described, the situation of the parathyroid glands preserved was correlated with extensive CLND. The coefficient of correlation was -0.18 , indicating that more extensive CLND might lead to a lower number and reduced blood supply of preserved parathyroid glands. Pearson chi-square test showed a statistically significant difference $(\mathrm{P}<0.05)$.

\section{Discussion}

Surgical complications following thyroidectomy are seen as hypocalcemia, voice change, vocal cord dysfunction, 
hematoma, and so on. Among these, hypocalcemia is probably more difficult to prevent due to extent of oncologic surgical resection and parathyroid glands preservation (14). Despite capsular dissection technique and parathyroid autotransplantation being performed, hypocalcemia in our study was $48.1 \%$, which was similar to that of other recent reports $(2,3,15)$.

Participants' clinicopathologic characteristics, perioperative serum Cs, and PTH level are among the main predictive factors of postoperative hypocalcemia $(16,17)$. In our experience, various intraoperative factors, such as mechanical and thermal injury, de-vascularization, and incidental excision of parathyroid glands, are more closely related to hypocalcemia. Because of strong relationship between hypocalcemia and parathyroid glands preserved, conventional thyroid surgical strategy recommends the systemic identification and preservation of as many parathyroid glands as possible. As research continues, the requirement to routinely identify all parathyroid glands has been questioned and remains controversial. Time-consuming, meticulous dissection for preservation of functional parathyroid glands is always destined to fail sometimes in CLND, either because of thrombosis of the parathyroid artery or parathyroid capsule edema (18). In this instance, an appropriate number of parathyroid glands preserved is recommended. Some authors reported a higher incidence of postoperative hypocalcemia when less than 3 parathyroid glands were identified and preserved (19), while others reported that less than 2 parathyroid glands identified during surgery was a risk of postoperative development (20). Furthermore, some authors recommended that preserving 1 parathyroid gland with an intact blood supply was enough (18).

In the current study, the preservation of parathyroid glands was categorized according to the number of parathyroid glands preserved in situ and autotransplanted. This classification was based on anatomy and our experience. In terms of anatomy, parathyroid glands are usually located extracapsularly on the posterior surface of the thyroid gland. The 2 superior parathyroids are usually located at the superior pole of the posterior thyroid gland near the cricothyroid junction. They have relatively fixed locations and are generally very apparent for quick identification. However, some of the inferior parathyroids are usually found in the lower pole of the thyroid gland and have high rates of anatomic variations. According to our experience, it is relatively easier to identify and preserve the 2 superior parathyroids and their blood supply than 2 inferior parathyroids. Therefore, we defined the boundary as a cut point at 2 parathyroids. Meanwhile, blood supplies are inherently more vulnerable to surgical trauma, which mainly affects the function of normal parathyroids (Figure 1). We aim to actively or passively autotransplant parathyroid glands which cannot be preserved in situ with good blood supplies. Therefore, in order to clarify the effectiveness of parathyroid autotransplantation, we set up research groups $\mathrm{E}$ and $\mathrm{F}$.

Our data showed that the variation of serum $\mathrm{Ca}$ level was primarily affected by PTH changes. Group F had the highest serum $\mathrm{Ca}$ and PTH level at any time after surgery, and the frequencies of hypocalcemia were lowest among others, which could prevent postoperative hypocalcemia effectively. We deem it optimal that 3-4 parathyroid glands are preserved during operation, regardless of whether by parathyroid autotransplantation. If only 1-2 parathyroid glands are preserved, it may lead to noticeably low postoperative serum $\mathrm{Ca}$, low serum $\mathrm{PTH}$, and the highest frequencies of hypocalcemia, especially in symptomatic hypocalcemia. However, in cases where preservation of 3-4 parathyroid glands fails, we can try our best to identify and autotransplant. In the present study, group E, with both only 1-2 parathyroid glands in situ plus at least 1 parathyroid autotransplantation, had higher postoperative serum $\mathrm{Ca}$ and PTH level, and a lower rate of hypocalcemia than group D. Furthermore, such differences were not statistically significant between group $\mathrm{E}$ and $\mathrm{F}$. Thus, we suggest that more than 2 parathyroid glands should be preserved in situ and plus at least 1 parathyroid autotransplantation should be performed during the operation.

The crucial risk factor for hypocalcemia is the surgical technique itself and the extent of operation, which is associated with preservation of parathyroids. More extensive surgical procedures could lead to hypocalcemia (11). However, use of thyroidectomy with a capsular dissection technique leads to significant differences slowly disappearing among total, subtotal, and partial thyroidectomy in hypocalcemia (12). For safety in DTC surgery, CLND is essential in clinically N1 patients. Even for cN0 patients, most surgeons prefer to conduct prophylactic CLND. However, during CLND, parathyroid glands are easily removed with the surrounding adipose tissue, but it is hard to distinguish the glands from the lymph nodes around them. Therefore, we view CLND as the main risk factor for unintentionally excising parathyroids during surgery for DTC. Our data indicate the correlation between the more extensive CLND performed and the fewer parathyroid preserved. The risk of hypocalcemia increased when TT combined with CLND, and the risk was even higher 
for bilateral CLND than unilateral CLND, as reported previously. However, the variations in postoperative serum $\mathrm{Ca}$ and PTH levels had no significant differences between groups A and B, while significant differences were observed between groups A and C. The only differences between group $\mathrm{B}$ and $\mathrm{C}$ were in $\mathrm{PTH}$ and $\mathrm{POD}>7$ serum $\mathrm{Ca}$. These results indicate that more attention should be paid to hypocalcemia when bilateral CLND is performed. This finding that unilateral or bilateral CLND may be associated with higher risk of hypocalcemia may assist in resolving the hesitation and debate surrounding CLND for DTC, particularly in pCLND for $\mathrm{cN} 0$, which is currently still controversial in guidelines around the world (21-23).

This study also indicated that routine $\mathrm{Ca}$ supplementation, which has been recommended to minimize the rate of hypocalcemia and shorten hospital stays, may not be necessary (24). Selectively predictive Ca supplementation may be a better choice (25). Patients should be administered $\mathrm{Ca}$ or vitamin $\mathrm{D}$ supplementation preemptively after TT combined with CLND, especially for bilateral CLND. If the surgeon fails to retain 1-2 parathyroids in situ during the operation, and cannot identify and autotransplant more parathyroids, predictive Ca supplementation is recommended.

The present study had some unavoidable limitations. First, the sample size of our study was relatively small and the study design was not randomized. Second, all operations were performed by different teams of surgeons within the same hospital and data was collected from a single institution. Thus, the conclusions cannot be assumed applicable to all surgeons or centers. Third, the evaluation of the status and viability of the parathyroid glands' blood circulation remains a subjective determination. Finally, several other unknown factors could influence the results despite much effort to minimize them. Therefore, further larger and well-designed studies are needed.

\section{Conclusions}

Performance of CLND in thyroid cancer surgery increases the difficulty of parathyroid function preservation. With the expansion of CLND range, postoperative parathyroid function will be affected, increasing the risk of postoperative hypocalcemia. Surgeons should strive to preserve parathyroid glands in situ, and autotransplantation should be carried out for ischemic or free parathyroids. When at least 1-2 parathyroid glands were reserved in situ plus at least 1 parathyroid gland autotransplantation, there was little effect on postoperative parathyroid function, and the incidence of hypocalcemia was low. If this is not achievable, patients should be given preventive Ca support treatment after operation.

\section{Acknowledgments}

Funding: This work was supported by following grants: Key research and development projects of science and Technology Department of Sichuan Province (2019YF0329); Scientific research project of Sichuan health and Family Planning Commission (No. 5960).

\section{Footnote}

Reporting Checklist: The authors have completed the STROBE reporting checklist. Available at http://dx.doi. org/10.21037/gs-21-121

Data Sharing Statement: Available at http://dx.doi. org/10.21037/gs-21-121

Conflicts of Interest: All authors have completed the ICMJE uniform disclosure form (available at http://dx.doi. org/10.21037/gs-21-121). The authors have no conflicts of interest to declare.

Ethical Statement: The authors are accountable for all aspects of the work in ensuring that questions related to the accuracy or integrity of any part of the work are appropriately investigated and resolved. The study was reviewed and approved by the Ethics committee of Sichuan Cancer Hospital. All samples were anonymized before analysis was performed, to guarantee the protection of privacy. All procedures performed in this study involving human participants were in accordance with the Declaration of Helsinki (as revised in 2013). Individual consent for this retrospective analysis was waived.

Open Access Statement: This is an Open Access article distributed in accordance with the Creative Commons Attribution-NonCommercial-NoDerivs 4.0 International License (CC BY-NC-ND 4.0), which permits the noncommercial replication and distribution of the article with the strict proviso that no changes or edits are made and the original work is properly cited (including links to both the formal publication through the relevant DOI and the license). See: https://creativecommons.org/licenses/by-nc-nd/4.0/. 


\section{References}

1. Ponce de León-Ballesteros G, Velázquez-Fernández D, Hernández-Calderón FJ, et al. Hypoparathyroidism After Total Thyroidectomy: Importance of the Intraoperative Management of the Parathyroid Glands. World J Surg 2019;43:1728-35.

2. Arman S, Vijendren A, Mochloulis G.The incidence of post-thyroidectomy hypocalcaemia: a retrospective single-centre audit. Ann R Coll Surg Engl 2019;101:273-8.

3. Giordano D, Valcavi R, Thompson GB, et al. Complications of central neck dissection in patients with papillary thyroid carcinoma: results of a study on 1087 patients and review of the literature. Thyroid 2012;22:911-7.

4. Gartland RM, Bloom JP, Parangi S, et al. A Long, Unnerving Road: Malpractice Claims Involving the Surgical Management of Thyroid and Parathyroid Disease. World J Surg 2019;43:2850-5.

5. Li Y, Jian $\mathrm{WH}$, Guo ZM, et al. A Meta-analysis of Carbon Nanoparticles for Identifying Lymph Nodes and Protecting Parathyroid Glands during Surgery. Otolaryngol Head Neck Surg 2015;152:1007-16.

6. Kim SW, Lee HS, Ahn YC, et al. Near-Infrared Autofluorescence Image-Guided Parathyroid Gland Mapping in Thyroidectomy.J Am Coll Surg 2018;226:165-72.

7. Abboud B, Sleilaty G, Braidy C, et al. Careful examination of thyroid specimen intraoperatively to reduce incidence of inadvertent parathyroidectomy during thyroid surgery. Arch Otolaryngol Head Neck Surg 2007;133:1105-10.

8. Song CM, Jung JH, Ji YB, et al. Relationship between hypoparathyroidism and the number of parathyroid glands preserved during thyroidectomy. World J Surg Oncol 2014;12:200.

9. Wang B, Zhu CR, Liu H, et al. The inferior parathyroid glands preserved in site recover faster than the superior parathyroid glands preserved in site after thyroid surgery for carcinoma. Medicine (Baltimore) 2020;99:e20886.

10. Su A, Gong Y, Wu W, et al. Does the number of parathyroid glands autotransplanted affect the incidence of hypoparathyroidism and recovery of parathyroid function? Surgery 2018. [Epub ahead of print]. doi: 10.1016/ j.surg.2017.12.025.

11. Su A, Wang B, Gong $Y$, et al. Risk factors of hypoparathyroidism following total thyroidectomy with central lymph node dissection. Medicine (Baltimore) 2017;96:e8162.

12. Su A, Gong Y, Wei T, et al. A new classification of parathyroid glands to evaluate in situ preservation or autotransplantation during thyroid surgery. Medicine (Baltimore) 2018;97:e13231.

13. Delogu D, Pisano IP, Pala C, et al. Prophylactic central neck lymphadenectomy in high risk patients with $\mathrm{T} 1$ or T2 papillary thyroid carcinoma: is it useful? Ann Ital Chir 2014;85:225-9.

14. Weiss A, Parina RP, Tang JA, et al. Outcomes of thyroidectomy from a large California state database. Am J Surg 2015;210:1170-6.

15. Ahn SV, Lee JH, Bove-Fenderson, et al. Incidence of Hypoparathyroidism After Thyroid Cancer Surgery in South Korea, 2007-2016. JAMA 2019;322:2441-3.

16. Del Río L, Castro A, Bernáldez R, et al. Parathyroid hormone as a predictor of post-thyroidectomy hypocalcemia. Acta Otorrinolaringol Esp 2011;62:265-73.

17. Sands NB, Payne RJ, Côté V, et al. Female gender as a risk factor for transient post-thyroidectomy hypocalcemia. Otolaryngol Head Neck Surg 2011;145:561-4.

18. Zhu J, Tian W, Xu Z, et al. Expert consensus statement on parathyroid protection in thyroidectomy. Ann Transl Med 2015;3:230.

19. Pattou F, Combemale F, Fabre S, et al. Hypocalcemia following thyroid surgery: incidence and prediction of outcome. World J Surg 1998;22:718-24.

20. Thomusch O, Machens A, Sekulla C, et al. The impact of surgical technique on postoperative hypoparathyroidism in bilateral thyroid surgery: a multivariate analysis of 5846 consecutive patients. Surgery 2003;133:180-5.

21. Haugen BR, Alexander EK, Bible KC, et al. 2015 American Thyroid Association Management Guidelines for Adult Patients with Thyroid Nodules and Differentiated Thyroid Cancer: The American Thyroid Association Guidelines Task Force on Thyroid Nodules and Differentiated Thyroid Cancer. Thyroid 2016;26:1-133.

22. Pacini F, Castagna MG, Brilli L, et al. Thyroid cancer: ESMO Clinical Practice Guidelines for diagnosis, treatment and follow-up. Ann Oncol 2010;21:v214-9.

23. Takami H, Ito $Y$, Okamoto $T$, et al. Revisiting the guidelines issued by the Japanese Society of Thyroid Surgeons and Japan Association of Endocrine Surgeons: a gradual move towards consensus between Japanese and western practice in the management of thyroid carcinoma. 
World J Surg 2014;38:2002-10.

24. Grodski S, Serpell J. Evidence for the role of perioperative PTH measurement after total thyroidectomy as a predictor of hypocalcemia. World J Surg 2008;32:1367-73.

Cite this article as: Sun R, Sheng J, Zhou Y, Zhou Y, Cai Y, Shui C, Zeng D, Jiang J, Li R, Wang X, Zhu J, Li C. Relationship between the extent of central node dissection and parathyroid function preservation in thyroid cancer surgery. Gland Surg 2021;10(3):1093-1103. doi: 10.21037/gs-21-121
25. Sun R, Zhang J, Zhang F, et al. Selectively predictive calcium supplementation using NCCN risk stratification system after thyroidectomy with differentiated thyroid cancer. Int J Clin Exp Med 2015;8:21939-46. 